Supplement of Web Ecol., 17, 69-77, 2017

https://doi.org/10.5194/we-17-69-2017-supplement

(C) Author(s) 2017. This work is distributed under

the Creative Commons Attribution 4.0 License.

(c) (i)

Supplement of

\title{
Modelling plant invasion pathways in protected areas under climate change: implication for invasion management
}

Chun-Jing Wang et al.

Correspondence to: Zhi-Xiang Zhang (zxzhang@bjfu.edu.cn)

The copyright of individual parts of the supplement might differ from the CC BY 4.0 License. 


\section{Supporting information}

2 Table S1 Environmental variables used. Environmental variables were used as

3 environmental layers for modelling the habitat suitability of IPS using Maxent; C of

$4 \quad$ V represents the coefficient of variation; SD: standard deviation.

\begin{tabular}{llll}
\hline Code & Environmental variables & Unit & References \\
\hline Bio1 & Annual mean temperature & ${ }^{\circ} \mathrm{C}$ & Average climate \\
Bio4 & Temperature seasonality & SD & Standard deviation of climate \\
Bio12 & Annual precipitation & $\mathrm{mm}$ & Average climate \\
Bio15 & Precipitation seasonality & $\mathrm{C}$ of V & Standard deviation of climate \\
\hline
\end{tabular}


Table S2. IPS, AUC values, and the results of the Maxent jackknife test. Codes as described in Table S1.

\begin{tabular}{|c|c|c|c|c|c|c|c|c|}
\hline Name & Type & Record & $\mathrm{AUC}_{\text {Training }}$ & $\mathrm{AUC}_{\text {Test }}$ & Bio 1 & Bio 4 & Bio 12 & Bio 15 \\
\hline Abelmoschus moschatus & Herb & 482 & 0.929 & 0.926 & 22.431 & 18.693 & 58.012 & 0.863 \\
\hline Abrus precatorius & Shrub & 1,059 & 0.915 & 0.914 & 49.521 & 15.270 & 31.103 & 4.105 \\
\hline Acacia farnesiana & Tree & 1,823 & 0.865 & 0.863 & 62.807 & 19.586 & 11.756 & 5.850 \\
\hline Acacia longifolia & Tree & 834 & 0.957 & 0.957 & 40.648 & 52.378 & 5.372 & 1.603 \\
\hline Acacia mangium & Tree & 198 & 0.946 & 0.941 & 29.381 & 30.111 & 40.046 & 0.462 \\
\hline Acacia mearnsii & Tree & 597 & 0.964 & 0.963 & 42.284 & 39.702 & 15.543 & 2.471 \\
\hline Acacia melanoxylon & Tree & 1,199 & 0.940 & 0.940 & 33.911 & 47.429 & 16.152 & 2.508 \\
\hline Acacia nilotica & Tree & 662 & 0.924 & 0.921 & 31.944 & 36.808 & 21.472 & 9.776 \\
\hline Acacia retinodes & Tree & 282 & 0.977 & 0.972 & 48.693 & 43.250 & 5.451 & 2.606 \\
\hline Acacia saligna & Tree & 796 & 0.953 & 0.953 & 54.852 & 37.958 & 6.678 & 0.512 \\
\hline Acanthophora spicifera & Alga & 130 & 0.938 & 0.929 & 38.912 & 49.787 & 7.175 & 4.126 \\
\hline Acanthus mollis & Herb & 332 & 0.970 & 0.969 & 48.354 & 32.692 & 16.947 & 2.006 \\
\hline Acer ginnala & Tree & 116 & 0.966 & 0.961 & 53.601 & 15.274 & 26.814 & 4.312 \\
\hline Acer platanoides & Tree & 6,061 & 0.815 & 0.815 & 50.635 & 17.913 & 25.467 & 5.986 \\
\hline Acroptilon repens & Herb & 266 & 0.939 & 0.935 & 57.880 & 26.211 & 12.396 & 3.514 \\
\hline Adenanthera pavonina & Tree & 158 & 0.943 & 0.939 & 20.497 & 20.115 & 57.249 & 2.140 \\
\hline Aegilops triuncialis & Herb & 1,405 & 0.921 & 0.921 & 68.425 & 16.801 & 11.970 & 2.804 \\
\hline Agapanthus praecox & Herb & 161 & 0.987 & 0.986 & 33.998 & 44.572 & 15.198 & 6.232 \\
\hline Agave americana & Herb & 460 & 0.949 & 0.946 & 55.494 & 32.979 & 8.219 & 3.308 \\
\hline Ageratina adenophora & Shrub & 398 & 0.968 & 0.967 & 44.351 & 42.625 & 11.183 & 1.841 \\
\hline Ageratina riparia & Herb & 192 & 0.984 & 0.982 & 34.915 & 21.486 & 35.517 & 8.082 \\
\hline Ageratum conyzoides & Herb & 1,236 & 0.886 & 0.884 & 20.993 & 42.884 & 35.289 & 0.834 \\
\hline
\end{tabular}




\begin{tabular}{llrrrrrrr}
\hline Agrostis capillaris & Herb & 12,366 & 0.723 & 0.723 & 42.640 & 21.103 & 31.631 & 4.627 \\
Agrostis gigantea & Herb & 6,746 & 0.788 & 0.788 & 59.094 & 19.739 & 19.637 & 1.529 \\
Ailanthus altissima & Tree & 1,473 & 0.915 & 0.914 & 67.887 & 13.939 & 11.057 & 7.118 \\
Akebia quinata & Vine & 153 & 0.979 & 0.977 & 33.452 & 16.548 & 46.931 & 3.069 \\
Albizia julibrissin & Tree & 273 & 0.956 & 0.953 & 53.047 & 14.343 & 32.158 & 0.452 \\
Albizia lebbeck & Tree & 394 & 0.919 & 0.915 & 53.218 & 19.065 & 24.039 & 3.678 \\
Aleurites moluccana & Tree & 112 & 0.944 & 0.937 & 17.036 & 35.628 & 45.361 & 1.975 \\
Alliaria petiolata & Herb & 6,086 & 0.813 & 0.813 & 49.737 & 14.539 & 25.098 & 10.627 \\
Alnus glutinosa & Tree & 8,732 & 0.770 & 0.769 & 45.339 & 19.665 & 28.944 & 6.052 \\
Alternanthera philoxeroides & Aquatic Plant & 228 & 0.955 & 0.951 & 32.770 & 15.480 & 33.932 & 17.819 \\
Alternanthera sessilis & Herb & 624 & 0.886 & 0.883 & 36.994 & 37.033 & 24.005 & 1.969 \\
Ambrosia artemisiifolia & Herb & 2,604 & 0.862 & 0.861 & 47.055 & 7.699 & 30.281 & 14.965 \\
Ammophila arenaria & Herb & 1,683 & 0.922 & 0.922 & 41.162 & 41.845 & 14.345 & 2.649 \\
Ampelopsis brevipedunculata & Vine & 201 & 0.975 & 0.972 & 13.630 & 24.011 & 59.889 & 2.471 \\
Andropogon gayanus & Herb & 536 & 0.934 & 0.932 & 40.415 & 40.278 & 11.856 & 7.451 \\
Andropogon virginicus & Herb & 607 & 0.941 & 0.939 & 40.447 & 9.972 & 44.399 & 5.184 \\
Angiopteris evecta & Fern & 97 & 0.944 & 0.939 & 18.917 & 13.208 & 67.343 & 0.532 \\
Annona glabra & Tree & 280 & 0.953 & 0.950 & 19.133 & 38.346 & 38.547 & 3.974 \\
Annona squamosa & Tree & 182 & 0.927 & 0.921 & 23.216 & 41.478 & 31.450 & 3.856 \\
Anredera cordifolia & Vine & 381 & 0.961 & 0.960 & 49.387 & 33.839 & 13.055 & 3.719 \\
Antigonon leptopus & Vine & 402 & 0.902 & 0.895 & 46.329 & 39.923 & 9.427 & 4.322 \\
Archontophoenix cunninghamiana & Tree & 176 & 0.989 & 0.986 & 24.314 & 31.865 & 32.998 & 10.823 \\
Ardisia crenata & Shrub & 229 & 0.969 & 0.967 & 9.081 & 14.855 & 68.654 & 7.410 \\
Arundo donax & Herb & 1,280 & 0.893 & 0.891 & 67.193 & 22.487 & 8.652 & 1.669 \\
Asparagus densiflorus & Herb & 121 & 0.954 & 0.947 & 29.453 & 47.713 & 13.337 & 9.497 \\
\hline & & & & & & &
\end{tabular}




\begin{tabular}{llrrrrrrr}
\hline Asparagus officinalis & Herb & 3,708 & 0.856 & 0.856 & 55.900 & 13.832 & 11.958 & 18.310 \\
Asystasia gangetica & Herb & 526 & 0.917 & 0.914 & 15.208 & 67.199 & 14.908 & 2.685 \\
Austroeupatorium inulifolium & Herb & 119 & 0.977 & 0.973 & 21.616 & 70.422 & 4.597 & 3.365 \\
Azolla pinnata & Aquatic Plant & 379 & 0.944 & 0.940 & 35.352 & 36.581 & 17.070 & 10.997 \\
Bacopa monnieri & Herb & 595 & 0.905 & 0.900 & 52.793 & 28.273 & 12.138 & 6.796 \\
Bambusa vulgaris & Herb & 152 & 0.916 & 0.906 & 10.871 & 61.446 & 25.350 & 2.333 \\
Begonia cucullata & Herb & 136 & 0.947 & 0.941 & 34.556 & 16.891 & 45.252 & 3.301 \\
Bellis perennis & Herb & 7,632 & 0.788 & 0.787 & 40.742 & 33.390 & 23.138 & 2.730 \\
Berberis darwinii & Tree & 313 & 0.983 & 0.982 & 35.878 & 46.356 & 11.987 & 5.779 \\
Berberis thunbergii & Shrub & 895 & 0.944 & 0.944 & 52.762 & 11.853 & 23.712 & 11.673 \\
Bidens pilosa & Herb & 2,356 & 0.847 & 0.846 & 50.435 & 21.144 & 27.146 & 1.275 \\
Bothriochloa pertusa & Herb & 325 & 0.926 & 0.923 & 55.793 & 10.789 & 27.130 & 6.288 \\
Brassica elongata & Herb & 210 & 0.965 & 0.962 & 42.667 & 28.770 & 8.467 & 20.096 \\
Brassica tournefortii & Herb & 1,712 & 0.911 & 0.910 & 61.237 & 23.444 & 11.491 & 3.828 \\
Bromus inermis & Herb & 4,361 & 0.826 & 0.825 & 64.766 & 18.369 & 13.469 & 3.396 \\
Bromus rubens & Herb & 2,692 & 0.882 & 0.881 & 70.893 & 22.782 & 5.943 & 0.382 \\
Bromus tectorum & Herb & 4,759 & 0.820 & 0.820 & 70.181 & 26.200 & 3.505 & 0.114 \\
Buddleja davidii & Shrub & 2,423 & 0.901 & 0.900 & 42.827 & 16.782 & 20.096 & 20.295 \\
Buddleja madagascariensis & Vine & 112 & 0.980 & 0.976 & 33.344 & 34.433 & 29.200 & 3.023 \\
Butomus umbellatus & Aquatic Plant & 3,207 & 0.877 & 0.877 & 43.134 & 17.757 & 21.138 & 17.972 \\
Cabomba caroliniana & Aquatic Plant & 218 & 0.958 & 0.955 & 19.815 & 20.604 & 47.068 & 12.512 \\
Caesalpinia decapetala & Tree & 252 & 0.958 & 0.955 & 44.576 & 4.500 & 40.962 & 9.963 \\
Calluna vulgaris & Herb & 12,530 & 0.721 & 0.721 & 39.715 & 23.134 & 31.872 & 5.279 \\
Camelina sativa & Herb & 1,548 & 0.921 & 0.920 & 49.469 & 19.197 & 15.342 & 15.993 \\
Campuloclinium macrocephalum & Herb & 151 & 0.978 & 0.974 & 30.076 & 33.290 & 28.808 & 7.826 \\
\hline & & & & & & &
\end{tabular}




\begin{tabular}{llrrrrrrr}
\hline Canna indica & Herb & 674 & 0.907 & 0.904 & 19.548 & 38.563 & 37.298 & 4.592 \\
Cardamine flexuosa & Herb & 5,446 & 0.822 & 0.822 & 33.657 & 30.089 & 33.430 & 2.824 \\
Cardiospermum grandiflorum & Vine & 428 & 0.937 & 0.934 & 29.007 & 34.584 & 26.039 & 10.369 \\
Carduus nutans & Herb & 3,865 & 0.859 & 0.859 & 55.670 & 11.815 & 13.684 & 18.831 \\
Carpobrotus edulis & Succulent & 433 & 0.968 & 0.967 & 43.148 & 51.273 & 4.362 & 1.217 \\
Castilla elastica & Tree & 194 & 0.956 & 0.951 & 10.242 & 31.375 & 54.402 & 3.982 \\
Casuarina equisetifolia & Tree & 528 & 0.916 & 0.913 & 30.216 & 38.459 & 29.342 & 1.983 \\
Caulerpa taxifolia & Alga & 111 & 0.941 & 0.928 & 19.663 & 59.791 & 9.723 & 10.823 \\
Cecropia peltata & Tree & 366 & 0.942 & 0.940 & 2.232 & 53.717 & 34.052 & 9.999 \\
Cecropia schreberiana & Tree & 379 & 0.942 & 0.940 & 1.615 & 54.552 & 33.449 & 10.383 \\
Cedrela odorata & Tree & 517 & 0.919 & 0.916 & 10.107 & 56.170 & 32.339 & 1.384 \\
Celastrus orbiculatus & Vine & 328 & 0.973 & 0.972 & 30.923 & 18.581 & 48.586 & 1.911 \\
Cenchrus ciliaris & Herb & 1,858 & 0.877 & 0.876 & 61.520 & 15.758 & 20.173 & 2.549 \\
Cenchrus echinatus & Herb & 1,162 & 0.856 & 0.853 & 72.286 & 11.483 & 13.049 & 3.182 \\
Cenchrus polystachios & Herb & 104 & 0.938 & 0.933 & 34.412 & 42.759 & 11.595 & 11.234 \\
Centaurea diffusa & Herb & 308 & 0.960 & 0.959 & 54.713 & 39.933 & 3.823 & 1.531 \\
Centaurea melitensis & Herb & 2,593 & 0.881 & 0.880 & 60.702 & 32.019 & 6.444 & 0.835 \\
Centaurea solstitialis & Herb & 1,445 & 0.918 & 0.918 & 51.308 & 40.160 & 8.018 & 0.513 \\
Cerastium fontanum & Herb & 11,681 & 0.728 & 0.728 & 44.356 & 24.378 & 28.480 & 2.786 \\
Ceratophyllum demersum & Aquatic Plant & 2,064 & 0.831 & 0.829 & 55.671 & 8.534 & 29.414 & 6.381 \\
Cestrum nocturnum & Shrub & 433 & 0.941 & 0.938 & 18.205 & 34.172 & 43.665 & 3.958 \\
Cestrum parqui & Shrub & 399 & 0.962 & 0.960 & 34.925 & 51.230 & 9.468 & 4.378 \\
Chromolaena odorata & Herb & 673 & 0.912 & 0.909 & 12.124 & 48.250 & 36.387 & 3.239 \\
Chrysanthemoides monilifera & Herb & 341 & 0.978 & 0.978 & 40.042 & 51.332 & 4.684 & 3.943 \\
Chrysobalanus icaco & Tree & 499 & 0.949 & 0.947 & 15.401 & 43.757 & 38.429 & 2.414 \\
\hline
\end{tabular}




\begin{tabular}{llrrrrrrr}
\hline Cinchona pubescens & Tree & 223 & 0.976 & 0.975 & 26.103 & 68.126 & 4.531 & 1.240 \\
Cinnamomum camphora & Tree & 416 & 0.958 & 0.956 & 34.968 & 18.077 & 40.373 & 6.581 \\
Cirsium arvense & Herb & 10,017 & 0.747 & 0.747 & 58.700 & 22.390 & 15.046 & 3.863 \\
Cirsium vulgare & Herb & 11,024 & 0.734 & 0.734 & 48.442 & 27.659 & 19.833 & 4.066 \\
Clematis terniflora & Vine & 366 & 0.970 & 0.969 & 30.498 & 15.702 & 51.485 & 2.315 \\
Clematis vitalba & Vine & 4,525 & 0.848 & 0.848 & 40.639 & 30.648 & 12.044 & 16.670 \\
Clidemia hirta & Shrub & 614 & 0.939 & 0.937 & 3.250 & 57.038 & 34.851 & 4.862 \\
Coccinia grandis & Vine & 150 & 0.918 & 0.911 & 16.778 & 79.042 & 3.276 & 0.903 \\
Colubrina asiatica & Shrub & 258 & 0.949 & 0.945 & 25.312 & 21.255 & 52.932 & 0.501 \\
Commelina benghalensis & Herb & 572 & 0.902 & 0.898 & 50.904 & 26.910 & 19.546 & 2.639 \\
Coronilla varia & Herb & 356 & 0.949 & 0.946 & 56.155 & 18.778 & 18.584 & 6.482 \\
Cortaderia jubata & Herb & 148 & 0.980 & 0.978 & 39.924 & 56.140 & 2.617 & 1.319 \\
Cortaderia selloana & Herb & 671 & 0.952 & 0.951 & 45.248 & 49.411 & 4.682 & 0.660 \\
Cotinus coggygria & Tree & 228 & 0.966 & 0.964 & 39.328 & 32.796 & 25.594 & 2.282 \\
Crassula helmsii & Aquatic Plant & 921 & 0.957 & 0.956 & 33.369 & 37.732 & 4.599 & 24.300 \\
Cryptostegia grandiflora & Vine & 311 & 0.949 & 0.944 & 44.704 & 25.414 & 19.057 & 10.825 \\
Cryptostegia madagascariensis & Vine & 153 & 0.944 & 0.940 & 27.722 & 43.413 & 14.549 & 14.316 \\
Cupaniopsis anacardioides & Tree & 535 & 0.963 & 0.962 & 26.634 & 31.259 & 37.002 & 5.106 \\
Cyathea cooperi & Fern & 248 & 0.977 & 0.975 & 24.562 & 34.880 & 33.768 & 6.790 \\
Cynara cardunculus & Herb & 457 & 0.961 & 0.960 & 54.565 & 36.028 & 9.166 & 0.241 \\
Cynodon dactylon & Herb & 5,874 & 0.773 & 0.772 & 63.184 & 28.544 & 7.730 & 0.542 \\
Cynoglossum officinale & Herb & 2,814 & 0.888 & 0.888 & 51.003 & 22.056 & 12.723 & 14.218 \\
Cyperus rotundus & Herb & 2,307 & 0.828 & 0.827 & 72.768 & 10.682 & 13.319 & 3.231 \\
Cytisus scoparius & Shrub & 6,501 & 0.808 & 0.808 & 43.223 & 35.920 & 19.293 & 1.564 \\
Cytisus striatus & Shrub & 338 & 0.971 & 0.970 & 46.185 & 38.549 & 13.707 & 1.559 \\
\hline
\end{tabular}




\begin{tabular}{llrrrrrrr}
\hline Delairea odorata & Vine & 339 & 0.976 & 0.975 & 39.275 & 54.379 & 5.342 & 1.005 \\
Dichrostachys cinerea & Tree & 689 & 0.924 & 0.922 & 21.362 & 48.096 & 15.147 & 15.395 \\
Dioscorea bulbifera & Herb & 677 & 0.926 & 0.923 & 33.232 & 7.909 & 52.128 & 6.731 \\
Dipogon lignosus & Vine & 264 & 0.980 & 0.979 & 37.835 & 55.907 & 4.085 & 2.174 \\
Duchesnea indica & Herb & 780 & 0.940 & 0.938 & 56.658 & 7.684 & 31.054 & 4.604 \\
Egeria densa & Aquatic Plant & 373 & 0.933 & 0.928 & 54.120 & 16.575 & 24.398 & 4.907 \\
Eichhornia crassipes & Aquatic Plant & 797 & 0.869 & 0.866 & 44.198 & 15.838 & 37.633 & 2.330 \\
Elaeagnus angustifolia & Tree & 669 & 0.916 & 0.914 & 70.522 & 25.055 & 3.147 & 1.277 \\
Elaeagnus pungens & Shrub & 101 & 0.985 & 0.981 & 29.573 & 19.556 & 46.266 & 4.605 \\
Elaeagnus umbellata & Tree & 408 & 0.959 & 0.958 & 49.943 & 3.895 & 45.190 & 0.972 \\
Elaeis guineensis & Palm & 139 & 0.961 & 0.958 & 21.002 & 41.314 & 30.440 & 7.244 \\
Elephantopus mollis & Herb & 783 & 0.923 & 0.920 & 12.341 & 33.120 & 53.895 & 0.644 \\
Elodea canadensis & Aquatic Plant & 1,248 & 0.911 & 0.910 & 51.383 & 11.145 & 20.262 & 17.211 \\
Epipremnum pinnatum & Vine & 179 & 0.934 & 0.928 & 7.703 & 7.770 & 80.750 & 3.778 \\
Erigeron karvinskianus & Herb & 1,018 & 0.928 & 0.927 & 43.910 & 34.527 & 20.456 & 1.107 \\
Eriobotrya japonica & Tree & 302 & 0.938 & 0.935 & 64.629 & 11.729 & 19.578 & 4.065 \\
Erodium cicutarium & Herb & 9,366 & 0.748 & 0.748 & 55.175 & 40.455 & 1.983 & 2.387 \\
Eugenia uniflora & Tree & 270 & 0.935 & 0.931 & 33.144 & 11.626 & 47.898 & 7.332 \\
Euonymus fortunei & Vine & 185 & 0.971 & 0.969 & 48.151 & 10.821 & 40.254 & 0.775 \\
Eupatorium cannabinum & Herb & 5,581 & 0.826 & 0.826 & 42.626 & 30.221 & 17.166 & 9.987 \\
Euphorbia esula & Herb & 2,694 & 0.886 & 0.886 & 49.077 & 17.847 & 18.569 & 14.507 \\
Ficus rubiginosa & Tree & 653 & 0.958 & 0.957 & 29.427 & 36.855 & 29.261 & 4.457 \\
Flacourtia indica & Tree & 260 & 0.930 & 0.924 & 23.327 & 35.169 & 23.746 & 17.758 \\
Flemingia strobilifera & Shrub & 119 & 0.950 & 0.945 & 10.886 & 5.171 & 80.664 & 3.278 \\
Fragaria vesca & Herb & 10,390 & 0.743 & 0.743 & 45.522 & 19.243 & 32.756 & 2.480 \\
\hline & & & & & &
\end{tabular}




\begin{tabular}{llrrrrrrr}
\hline Frangula alnus & Shrub & 5,653 & 0.821 & 0.821 & 44.062 & 25.375 & 25.527 & 5.037 \\
Fuchsia boliviana & Tree & 104 & 0.981 & 0.978 & 34.438 & 51.830 & 9.630 & 4.101 \\
Fuchsia magellanica & Vine & 845 & 0.960 & 0.960 & 37.542 & 40.235 & 21.506 & 0.717 \\
Genista monspessulana & Shrub & 645 & 0.958 & 0.957 & 50.950 & 37.771 & 10.962 & 0.317 \\
Glyceria maxima & Aquatic Plant & 4,565 & 0.846 & 0.846 & 44.556 & 16.830 & 22.099 & 16.515 \\
Grevillea robusta & Tree & 299 & 0.959 & 0.957 & 53.089 & 23.855 & 16.632 & 6.424 \\
Gunnera tinctoria & Herb & 255 & 0.986 & 0.986 & 36.327 & 43.677 & 17.266 & 2.731 \\
Haematoxylum campechianum & Tree & 219 & 0.975 & 0.974 & 34.312 & 24.739 & 26.731 & 14.218 \\
Hedera helix & Vine & 7,454 & 0.791 & 0.791 & 46.648 & 32.544 & 18.916 & 1.892 \\
Hedychium coronarium & Herb & 309 & 0.946 & 0.943 & 12.687 & 16.551 & 69.689 & 1.073 \\
Hedychium flavescens & Herb & 121 & 0.979 & 0.975 & 30.314 & 31.501 & 33.419 & 4.767 \\
Hedychium gardnerianum & Herb & 105 & 0.986 & 0.984 & 27.400 & 36.385 & 31.871 & 4.345 \\
Heliotropium curassavicum & Herb & 1,642 & 0.856 & 0.854 & 60.256 & 16.238 & 18.180 & 5.326 \\
Heracleum mantegazzianum & Herb & 3,279 & 0.879 & 0.879 & 44.306 & 21.632 & 19.902 & 14.160 \\
Heteropogon contortus & Herb & 2,595 & 0.848 & 0.847 & 49.502 & 28.480 & 14.555 & 7.463 \\
Hieracium aurantiacum & Herb & 1,563 & 0.926 & 0.926 & 42.534 & 19.564 & 26.047 & 11.855 \\
Hieracium floribundum & Herb & 195 & 0.976 & 0.975 & 38.433 & 10.048 & 27.031 & 24.488 \\
Hieracium pilosella & Herb & 5,058 & 0.835 & 0.835 & 37.220 & 38.703 & 19.816 & 4.262 \\
Holcus lanatus & Herb & 9,357 & 0.760 & 0.760 & 44.026 & 36.425 & 17.758 & 1.791 \\
Houttuynia cordata & Shrub & 209 & 0.963 & 0.956 & 25.346 & 20.203 & 50.271 & 4.180 \\
Hydrilla verticillata & Aquatic Plant & 547 & 0.907 & 0.904 & 39.052 & 17.063 & 41.147 & 2.739 \\
Hydrocharis morsus-ranae & Aquatic Plant & 2,762 & 0.892 & 0.892 & 43.492 & 13.180 & 23.875 & 19.453 \\
Hypericum perforatum & Herb & 8,903 & 0.763 & 0.763 & 52.781 & 20.873 & 24.049 & 2.298 \\
Hypnea musciformis & Alga & 96 & 0.928 & 0.916 & 48.368 & 28.605 & 16.234 & 6.793 \\
Hypochaeris radicata & Herb & 9,558 & 0.757 & 0.757 & 43.734 & 42.452 & 10.819 & 2.996 \\
\hline
\end{tabular}




\begin{tabular}{llrrrrrrr}
\hline Impatiens glandulifera & Herb & 4,709 & 0.840 & 0.840 & 50.125 & 19.187 & 25.246 & 5.443 \\
Impatiens walleriana & Herb & 265 & 0.945 & 0.942 & 23.688 & 28.171 & 43.554 & 4.586 \\
Imperata cylindrica & Herb & 2,091 & 0.858 & 0.857 & 63.103 & 12.959 & 21.384 & 2.554 \\
Ipomoea aquatica & Vine & 370 & 0.922 & 0.918 & 68.147 & 5.246 & 20.421 & 6.187 \\
Ipomoea cairica & Vine & 510 & 0.918 & 0.915 & 42.080 & 38.034 & 15.988 & 3.899 \\
Iris pseudacorus & Herb & 7,953 & 0.781 & 0.780 & 51.732 & 20.838 & 23.482 & 3.948 \\
Ischaemum polystachyum & Herb & 205 & 0.960 & 0.956 & 22.068 & 48.951 & 25.232 & 3.749 \\
Jatropha gossypiifolia & Herb & 403 & 0.912 & 0.908 & 27.959 & 54.574 & 14.810 & 2.657 \\
Juncus tenuis & Herb & 5,443 & 0.817 & 0.817 & 56.963 & 7.939 & 31.089 & 4.010 \\
Kalanchoe pinnata & Succulent & 381 & 0.934 & 0.931 & 16.292 & 28.581 & 51.765 & 3.362 \\
Lagarosiphon major & Aquatic Plant & 543 & 0.972 & 0.971 & 31.477 & 34.509 & 16.556 & 17.459 \\
Landoltia punctata & Aquatic Plant & 106 & 0.952 & 0.943 & 40.285 & 34.829 & 15.867 & 9.019 \\
Lantana camara & Shrub & 2,335 & 0.849 & 0.848 & 42.493 & 31.596 & 23.911 & 2.000 \\
Launaea intybacea & Shrub & 523 & 0.936 & 0.933 & 24.466 & 54.471 & 11.623 & 9.440 \\
Lepidium latifolium & Herb & 1,091 & 0.916 & 0.915 & 57.920 & 32.527 & 3.950 & 5.603 \\
Lepidium virginicum & Herb & 2,448 & 0.852 & 0.851 & 61.954 & 15.231 & 20.387 & 2.428 \\
Lespedeza cuneata & Herb & 392 & 0.967 & 0.965 & 25.449 & 23.126 & 50.353 & 1.072 \\
Leucaena leucocephala & Tree & 934 & 0.881 & 0.878 & 42.428 & 40.498 & 11.278 & 5.796 \\
Ligustrum lucidum & Tree & 441 & 0.950 & 0.948 & 64.420 & 10.278 & 21.906 & 3.396 \\
Ligustrum sinense & Tree & 485 & 0.961 & 0.960 & 44.302 & 13.260 & 38.952 & 3.487 \\
Ligustrum vulgare & Shrub & 5,600 & 0.826 & 0.826 & 44.747 & 26.620 & 15.152 & 13.481 \\
Limnocharis flava & Aquatic Plant & 190 & 0.926 & 0.921 & 13.124 & 55.101 & 30.864 & 0.911 \\
Linaria vulgaris & Herb & 8,279 & 0.772 & 0.771 & 48.118 & 19.971 & 27.955 & 3.956 \\
Litsea glutinosa & Tree & 487 & 0.962 & 0.960 & 15.531 & 7.400 & 50.450 & 26.619 \\
Lonicera japonica & Vine & 1,174 & 0.919 & 0.918 & 66.785 & 3.765 & 25.825 & 3.625 \\
\hline
\end{tabular}




\begin{tabular}{llrrrrrrr}
\hline Lonicera maackii & Shrub & 131 & 0.978 & 0.976 & 28.699 & 29.913 & 39.687 & 1.702 \\
Lotus corniculatus & Herb & 10,820 & 0.739 & 0.739 & 41.236 & 33.836 & 21.719 & 3.210 \\
Ludwigia peruviana & Aquatic Plant & 332 & 0.947 & 0.944 & 25.149 & 44.301 & 27.774 & 2.776 \\
Lupinus polyphyllus & Herb & 4,885 & 0.834 & 0.833 & 53.694 & 22.100 & 22.347 & 1.860 \\
Luzula campestris & Herb & 7,036 & 0.799 & 0.799 & 44.787 & 30.303 & 21.510 & 3.400 \\
Lygodium japonicum & Vine & 322 & 0.952 & 0.949 & 12.804 & 15.110 & 70.467 & 1.618 \\
Lygodium microphyllum & Fern & 663 & 0.927 & 0.925 & 35.828 & 22.445 & 40.140 & 1.588 \\
Lythrum salicaria & Aquatic Plant & 8,797 & 0.763 & 0.763 & 49.238 & 18.293 & 26.412 & 6.058 \\
Macfadyena unguis-cati & Vine & 676 & 0.913 & 0.911 & 27.765 & 34.807 & 34.139 & 3.289 \\
Melaleuca quinquenervia & Tree & 378 & 0.963 & 0.962 & 16.398 & 29.970 & 46.189 & 7.444 \\
Melia azedarach & Tree & 1,185 & 0.883 & 0.881 & 60.766 & 13.444 & 22.538 & 3.253 \\
Melilotus alba & Herb & 429 & 0.918 & 0.915 & 70.174 & 7.102 & 7.981 & 14.744 \\
Melinis minutiflora & Herb & 473 & 0.944 & 0.941 & 18.064 & 44.632 & 33.758 & 3.545 \\
Merremia tuberosa & Vine & 138 & 0.960 & 0.956 & 8.397 & 46.946 & 38.037 & 6.620 \\
Miconia calvescens & Tree & 258 & 0.947 & 0.942 & 10.935 & 70.454 & 16.063 & 2.548 \\
Microstegium vimineum & Herb & 282 & 0.973 & 0.971 & 21.543 & 21.738 & 55.751 & 0.969 \\
Mikania micrantha & Vine & 741 & 0.917 & 0.915 & 6.275 & 49.414 & 42.873 & 1.438 \\
Mimosa diplotricha & Vine & 190 & 0.938 & 0.933 & 16.487 & 29.574 & 53.307 & 0.633 \\
Mimosa pigra & Shrub & 930 & 0.896 & 0.893 & 20.933 & 61.909 & 16.593 & 0.565 \\
Mimosa pudica & Herb & 773 & 0.915 & 0.913 & 11.730 & 28.163 & 59.141 & 0.966 \\
Miscanthus sinensis & Herb & 395 & 0.949 & 0.946 & 25.686 & 21.763 & 51.851 & 0.700 \\
Montia fontana & Aquatic Plant & 6,878 & 0.789 & 0.789 & 43.054 & 29.227 & 25.455 & 2.265 \\
Morus alba & Tree & 910 & 0.898 & 0.896 & 81.039 & 4.892 & 11.157 & 2.911 \\
Myriophyllum aquaticum & Aquatic Plant & 828 & 0.937 & 0.936 & 49.132 & 18.660 & 20.724 & 11.485 \\
Myriophyllum heterophyllum & Aquatic Plant & 204 & 0.965 & 0.963 & 31.191 & 11.289 & 24.250 & 33.270 \\
\hline & & & & & & & &
\end{tabular}




\begin{tabular}{llrrrrrrr}
\hline Myriophyllum spicatum & Aquatic Plant & 4,538 & 0.837 & 0.837 & 46.413 & 21.751 & 19.855 & 11.981 \\
Najas minor & Aquatic Plant & 346 & 0.965 & 0.963 & 33.055 & 14.303 & 18.822 & 33.820 \\
Nassella neesiana & Herb & 409 & 0.959 & 0.957 & 41.575 & 52.691 & 4.202 & 1.532 \\
Nassella tenuissima & Herb & 177 & 0.968 & 0.965 & 39.677 & 50.854 & 7.335 & 2.134 \\
Nephrolepis cordifolia & Fern & 500 & 0.945 & 0.944 & 35.991 & 11.805 & 47.081 & 5.123 \\
Nicotiana glauca & Shrub & 1,539 & 0.902 & 0.901 & 67.317 & 22.282 & 10.159 & 0.242 \\
Nymphaea odorata & Aquatic Plant & 406 & 0.931 & 0.928 & 16.042 & 16.208 & 40.498 & 27.252 \\
Nymphoides peltata & Aquatic Plant & 1,215 & 0.942 & 0.942 & 43.596 & 12.377 & 17.059 & 26.968 \\
Ocimum gratissimum & Herb & 366 & 0.928 & 0.924 & 18.912 & 63.510 & 15.523 & 2.055 \\
Olea europaea & Tree & 1,779 & 0.907 & 0.907 & 70.055 & 22.269 & 6.683 & 0.993 \\
Onopordum acanthium & Herb & 3,407 & 0.873 & 0.873 & 38.483 & 25.350 & 10.378 & 25.789 \\
Opuntia monacantha & Tree & 139 & 0.957 & 0.950 & 45.245 & 33.873 & 10.246 & 10.636 \\
Opuntia stricta & Shrub & 674 & 0.951 & 0.949 & 39.034 & 33.261 & 9.792 & 17.913 \\
Oxalis corniculata & Herb & 5,330 & 0.803 & 0.802 & 48.728 & 29.357 & 19.014 & 2.901 \\
Oxalis latifolia & Herb & 560 & 0.936 & 0.934 & 44.158 & 42.880 & 11.695 & 1.268 \\
Oxalis pes-caprae & Herb & 1,090 & 0.938 & 0.937 & 66.371 & 29.483 & 3.767 & 0.379 \\
Oxycaryum cubense & Aquatic Plant & 211 & 0.932 & 0.926 & 29.369 & 42.583 & 23.101 & 4.948 \\
Paederia foetida & Vine & 287 & 0.959 & 0.954 & 12.787 & 17.446 & 64.277 & 5.491 \\
Panicum repens & Herb & 523 & 0.914 & 0.911 & 54.947 & 25.621 & 17.260 & 2.173 \\
Parthenium hysterophorus & Herb & 795 & 0.908 & 0.906 & 46.067 & 28.616 & 18.280 & 7.037 \\
Paspalum scrobiculatum & Herb & 1,496 & 0.884 & 0.882 & 36.639 & 27.839 & 34.742 & 0.779 \\
Paspalum urvillei & Herb & 614 & 0.947 & 0.945 & 54.788 & 16.105 & 25.584 & 3.523 \\
Paspalum vaginatum & Herb & 740 & 0.907 & 0.904 & 41.683 & 39.010 & 11.832 & 7.475 \\
Passiflora edulis & Vine & 569 & 0.935 & 0.933 & 25.348 & 20.633 & 49.246 & 4.773 \\
Passiflora foetida & Vine & 2,577 & 0.837 & 0.836 & 35.138 & 47.291 & 15.351 & 2.220 \\
\hline & & & & & &
\end{tabular}




\begin{tabular}{llrrrrrrr}
\hline Passiflora suberosa & Vine & 763 & 0.918 & 0.916 & 27.736 & 50.141 & 18.623 & 3.501 \\
Passiflora tarminiana & Vine & 111 & 0.986 & 0.984 & 38.798 & 46.184 & 11.104 & 3.914 \\
Paulownia tomentosa & Tree & 219 & 0.967 & 0.964 & 55.153 & 9.746 & 26.605 & 8.496 \\
Persicaria perfoliata L & Vine & 185 & 0.975 & 0.973 & 19.496 & 22.217 & 55.285 & 3.003 \\
Phalaris arundinacea & Herb & 10,360 & 0.741 & 0.740 & 50.972 & 15.966 & 28.660 & 4.402 \\
Phoenix canariensis & Tree & 185 & 0.964 & 0.961 & 60.376 & 34.256 & 2.701 & 2.667 \\
Phormium tenax & Shrub & 368 & 0.976 & 0.975 & 27.907 & 39.113 & 18.549 & 14.431 \\
Phragmites australis & Herb & 12,498 & 0.704 & 0.703 & 46.439 & 23.780 & 19.177 & 10.604 \\
Phymatosorus scolopendria & Herb & 493 & 0.940 & 0.938 & 6.402 & 46.179 & 43.221 & 4.199 \\
Physalis peruviana & Shrub & 945 & 0.924 & 0.922 & 35.063 & 31.203 & 29.331 & 4.403 \\
Pimenta dioica & Tree & 129 & 0.961 & 0.955 & 8.520 & 35.425 & 37.526 & 18.528 \\
Pinus caribaea & Tree & 140 & 0.965 & 0.960 & 3.790 & 35.334 & 48.103 & 12.773 \\
Pinus nigra & Tree & 3,117 & 0.883 & 0.883 & 45.318 & 24.348 & 12.053 & 18.281 \\
Pinus pinaster & Tree & 1,536 & 0.930 & 0.929 & 56.325 & 31.523 & 10.138 & 2.014 \\
Piper aduncum & Tree & 994 & 0.917 & 0.916 & 6.271 & 61.837 & 30.296 & 1.596 \\
Pistia stratiotes & Aquatic Plant & 648 & 0.885 & 0.882 & 26.234 & 28.220 & 42.051 & 3.495 \\
Pittosporum tenuifolium & Tree & 280 & 0.985 & 0.984 & 23.106 & 40.778 & 13.528 & 22.588 \\
Pittosporum undulatum & Tree & 597 & 0.967 & 0.967 & 41.454 & 38.635 & 17.476 & 2.435 \\
Pittosporum viridiflorum & Tree & 299 & 0.968 & 0.966 & 37.440 & 44.422 & 13.128 & 5.011 \\
Pluchea carolinensis & Shrub & 412 & 0.937 & 0.934 & 13.352 & 70.664 & 8.996 & 6.987 \\
Pluchea indica & Shrub & 125 & 0.959 & 0.953 & 46.077 & 25.697 & 25.325 & 2.902 \\
Poa annua & Herb & 13,678 & 0.702 & 0.702 & 45.435 & 30.674 & 21.254 & 2.637 \\
Polygala paniculata & Herb & 629 & 0.940 & 0.938 & 11.987 & 39.277 & 45.897 & 2.839 \\
Polygonum cuspidatum Sieb & Herb & 240 & 0.958 & 0.953 & 30.276 & 15.444 & 51.763 & 2.517 \\
Polysiphonia brodiei & Alga & 203 & 0.985 & 0.984 & 35.276 & 42.072 & 17.679 & 4.973 \\
\hline
\end{tabular}




\begin{tabular}{llrrrrrrr}
\hline Populus alba & Tree & 4,012 & 0.854 & 0.854 & 52.560 & 24.977 & 11.021 & 11.442 \\
Potamogeton crispus & Aquatic Plant & 3,987 & 0.845 & 0.845 & 40.862 & 23.446 & 18.207 & 17.485 \\
Potamogeton perfoliatus & Aquatic Plant & 4,843 & 0.827 & 0.827 & 40.325 & 23.293 & 25.617 & 10.766 \\
Prosopis glandulosa & Tree & 572 & 0.940 & 0.938 & 58.135 & 19.311 & 14.461 & 8.094 \\
Prosopis juliflora & Shrub & 278 & 0.902 & 0.895 & 29.991 & 50.090 & 13.724 & 6.195 \\
Psidium cattleianum & Tree & 107 & 0.962 & 0.953 & 30.886 & 21.844 & 38.417 & 8.853 \\
Psidium guajava & Tree & 961 & 0.900 & 0.898 & 21.117 & 44.130 & 32.875 & 1.878 \\
Psoralea pinnata & Shrub & 217 & 0.983 & 0.980 & 31.514 & 57.521 & 5.574 & 5.391 \\
Pteris cretica & Fern & 518 & 0.947 & 0.946 & 56.477 & 8.570 & 32.456 & 2.497 \\
Pyrus calleryana & Tree & 117 & 0.956 & 0.948 & 41.422 & 21.482 & 35.199 & 1.897 \\
Ranunculus ficaria & Herb & 7,132 & 0.796 & 0.796 & 41.111 & 31.865 & 24.677 & 2.347 \\
Rauvolfia vomitoria & Tree & 412 & 0.959 & 0.957 & 3.988 & 61.348 & 29.268 & 5.397 \\
Rhamnus alaternus & Tree & 1,132 & 0.939 & 0.939 & 50.035 & 36.678 & 12.460 & 0.827 \\
Rhamnus cathartica & Tree & 4,041 & 0.860 & 0.859 & 48.673 & 12.981 & 19.447 & 18.899 \\
Rhizophora mangle & Aquatic Plant & 467 & 0.924 & 0.921 & 33.223 & 58.288 & 4.956 & 3.533 \\
Rhododendron ponticum & Shrub & 1,712 & 0.930 & 0.930 & 31.759 & 39.488 & 13.972 & 14.781 \\
Rhus longipes & Tree & 103 & 0.970 & 0.966 & 12.010 & 55.232 & 19.435 & 13.324 \\
Ricinus communis & Tree & 1,405 & 0.851 & 0.850 & 45.414 & 43.632 & 9.773 & 1.181 \\
Robinia pseudoacacia & Tree & 4,777 & 0.835 & 0.835 & 59.505 & 13.219 & 15.087 & 12.189 \\
Rosa multiflora & Shrub & 1,006 & 0.930 & 0.929 & 60.267 & 5.393 & 27.336 & 7.005 \\
Rottboellia cochinchinensis & Herb & 498 & 0.898 & 0.894 & 31.527 & 44.225 & 23.346 & 0.901 \\
Rubus discolor & Vine & 859 & 0.954 & 0.954 & 44.749 & 22.026 & 10.437 & 22.788 \\
Rubus moluccanus & Vine & 522 & 0.960 & 0.959 & 23.662 & 21.346 & 46.786 & 8.206 \\
Rubus niveus & Shrub & 167 & 0.959 & 0.955 & 32.572 & 10.861 & 42.832 & 13.736 \\
Rubus phoenicolasius & Shrub & 231 & 0.980 & 0.979 & 52.672 & 4.767 & 32.027 & 10.535 \\
\hline & & & & & & &
\end{tabular}




\begin{tabular}{llrrrrrrr}
\hline Rubus pinnatus & Shrub & 141 & 0.982 & 0.980 & 29.314 & 50.356 & 14.198 & 6.132 \\
Rubus rosifolius & Tree & 592 & 0.960 & 0.959 & 35.145 & 15.128 & 43.643 & 6.084 \\
Rubus ulmifolius & Vine & 3,727 & 0.864 & 0.863 & 50.327 & 40.076 & 8.989 & 0.609 \\
Ruellia brevifolia & Herb & 150 & 0.948 & 0.942 & 26.087 & 44.620 & 22.915 & 6.378 \\
Rumex acetosella & Herb & 13,513 & 0.706 & 0.706 & 43.052 & 22.913 & 29.396 & 4.640 \\
Rumex crispus & Herb & 10,067 & 0.743 & 0.743 & 59.610 & 24.014 & 13.150 & 3.225 \\
Rumex obtusifolius & Herb & 7,533 & 0.787 & 0.786 & 49.372 & 24.579 & 22.538 & 3.511 \\
Sacciolepis indica & Herb & 757 & 0.925 & 0.923 & 22.051 & 7.295 & 65.588 & 5.066 \\
Sagina procumbens & Herb & 9,976 & 0.750 & 0.750 & 42.618 & 19.996 & 32.786 & 4.600 \\
Sagittaria platyphylla & Aquatic Plant & 157 & 0.977 & 0.974 & 50.315 & 11.198 & 20.803 & 17.684 \\
Sagittaria sagittifolia & Aquatic Plant & 3,197 & 0.878 & 0.878 & 40.952 & 14.462 & 23.712 & 20.874 \\
Salix babylonica & Tree & 850 & 0.935 & 0.934 & 60.331 & 18.248 & 12.587 & 8.834 \\
Salix cinerea & Tree & 7,545 & 0.788 & 0.788 & 45.942 & 15.680 & 30.706 & 7.673 \\
Salix humboldtiana & Tree & 324 & 0.918 & 0.913 & 8.232 & 73.912 & 13.409 & 4.447 \\
Salsola tragus & Shrub & 3,189 & 0.843 & 0.842 & 42.657 & 29.751 & 22.086 & 5.506 \\
Salvinia minima & Aquatic Plant & 167 & 0.942 & 0.936 & 29.546 & 14.946 & 45.656 & 9.852 \\
Salvinia molesta & Aquatic Plant & 268 & 0.929 & 0.921 & 45.997 & 26.382 & 21.802 & 5.819 \\
Samanea saman & Tree & 182 & 0.935 & 0.930 & 7.003 & 61.858 & 26.281 & 4.857 \\
Sansevieria hyacinthoides & Succulent & 118 & 0.966 & 0.962 & 18.650 & 50.328 & 17.946 & 13.075 \\
Sargassum muticum & Aquatic Plant & 328 & 0.975 & 0.974 & 43.068 & 36.509 & 17.419 & 3.004 \\
Scaevola sericea & Shrub & 144 & 0.958 & 0.954 & 20.767 & 16.574 & 58.393 & 4.266 \\
Schefflera actinophylla & Tree & 236 & 0.962 & 0.959 & 18.982 & 29.607 & 45.431 & 5.980 \\
Schinus terebinthifolius & Tree & 299 & 0.961 & 0.958 & 40.457 & 45.270 & 7.640 & 6.634 \\
Schismus arabicus & Herb & 436 & 0.947 & 0.945 & 47.142 & 15.554 & 34.778 & 2.527 \\
Schismus barbatus & Herb & 1,860 & 0.909 & 0.909 & 64.649 & 14.790 & 16.869 & 3.692 \\
\hline & & & & & & &
\end{tabular}




\begin{tabular}{llrrrrrrr}
\hline Sechium edule & Vine & 134 & 0.951 & 0.945 & 28.132 & 36.169 & 33.389 & 2.309 \\
Senecio angulatus & Herb & 149 & 0.986 & 0.985 & 45.861 & 40.642 & 9.666 & 3.831 \\
Senecio inaequidens & Shrub & 1,537 & 0.926 & 0.925 & 37.102 & 31.172 & 15.152 & 16.574 \\
Senecio jacobaea & Herb & 6,499 & 0.808 & 0.807 & 42.995 & 31.627 & 23.042 & 2.336 \\
Senecio squalidus & Herb & 1,128 & 0.947 & 0.947 & 30.226 & 37.939 & 8.908 & 22.928 \\
Senecio viscosus & Herb & 5,316 & 0.830 & 0.830 & 47.109 & 20.900 & 24.956 & 7.035 \\
Senecio vulgaris & Herb & 9,577 & 0.751 & 0.751 & 47.518 & 30.139 & 18.907 & 3.436 \\
Sesbania punicea & Shrub & 133 & 0.970 & 0.967 & 58.307 & 20.035 & 17.179 & 4.479 \\
Setaria verticillata & Herb & 2,886 & 0.858 & 0.858 & 41.261 & 43.164 & 9.299 & 6.276 \\
Solanum mauritianum & Tree & 470 & 0.965 & 0.964 & 38.861 & 34.103 & 23.058 & 3.978 \\
Solanum seaforthianum & Vine & 475 & 0.934 & 0.931 & 39.635 & 21.277 & 32.486 & 6.602 \\
Solanum sisymbriifolium & Herb & 286 & 0.935 & 0.932 & 24.516 & 33.186 & 37.451 & 4.847 \\
Solanum torvum & Shrub & 1,034 & 0.912 & 0.911 & 8.431 & 36.640 & 52.616 & 2.313 \\
Solidago canadensis & Herb & 4,881 & 0.825 & 0.825 & 62.821 & 11.827 & 19.298 & 6.054 \\
Sorghum halepense & Herb & 2,460 & 0.840 & 0.839 & 71.230 & 14.294 & 13.832 & 0.645 \\
Spartina alterniflora & Herb & 197 & 0.955 & 0.953 & 13.610 & 11.292 & 41.235 & 33.863 \\
Spartina anglica & Herb & 360 & 0.981 & 0.981 & 33.951 & 33.362 & 12.705 & 19.982 \\
Spartium junceum & Shrub & 979 & 0.940 & 0.939 & 54.451 & 36.157 & 9.370 & 0.022 \\
Spathodea campanulata & Tree & 243 & 0.927 & 0.922 & 6.006 & 70.954 & 21.338 & 1.702 \\
Spermacoce verticillata & Shrub & 677 & 0.914 & 0.911 & 7.742 & 64.707 & 25.115 & 2.436 \\
Sphagneticola trilobata & Herb & 458 & 0.942 & 0.939 & 15.255 & 26.974 & 53.802 & 3.970 \\
Spiraea japonica & Shrub & 533 & 0.949 & 0.947 & 44.417 & 28.207 & 26.659 & 0.717 \\
Stellaria alsine & Herb & 6,420 & 0.806 & 0.806 & 36.727 & 30.294 & 30.348 & 2.631 \\
Stellaria media & Herb & 12,825 & 0.710 & 0.710 & 43.497 & 28.496 & 24.518 & 3.489 \\
Striga asiatica & Herb & 289 & 0.921 & 0.916 & 21.309 & 39.235 & 26.074 & 13.382 \\
\hline
\end{tabular}




\begin{tabular}{llrrrrrrr}
\hline Syngonium podophyllum & Vine & 581 & 0.941 & 0.939 & 9.501 & 32.353 & 55.244 & 2.902 \\
Syzygium cumini & Tree & 151 & 0.921 & 0.911 & 24.922 & 34.017 & 37.014 & 4.047 \\
Syzygium jambos & Tree & 444 & 0.936 & 0.933 & 14.103 & 29.856 & 53.974 & 2.067 \\
Tamarix aphylla & Shrub & 266 & 0.934 & 0.930 & 66.647 & 6.298 & 25.104 & 1.952 \\
Tamarix parviflora & Tree & 288 & 0.951 & 0.948 & 75.300 & 16.361 & 5.366 & 2.972 \\
Tamarix ramosissima & Tree & 760 & 0.920 & 0.918 & 64.332 & 16.615 & 15.270 & 3.783 \\
Taraxacum officinale & Herb & 4,927 & 0.809 & 0.808 & 53.137 & 23.658 & 20.379 & 2.825 \\
Tecoma capensis & Vine & 215 & 0.961 & 0.958 & 45.206 & 41.750 & 9.162 & 3.882 \\
Tecoma stans & Tree & 620 & 0.900 & 0.896 & 40.371 & 44.599 & 8.925 & 6.104 \\
Terminalia catappa & Tree & 362 & 0.937 & 0.935 & 17.666 & 53.199 & 27.617 & 1.518 \\
Thevetia peruviana & Tree & 342 & 0.919 & 0.914 & 31.316 & 42.798 & 19.050 & 6.837 \\
Thunbergia grandiflora & Vine & 150 & 0.935 & 0.927 & 12.055 & 19.904 & 66.036 & 2.005 \\
Tithonia diversifolia & Shrub & 373 & 0.940 & 0.937 & 14.633 & 35.141 & 45.782 & 4.445 \\
Tradescantia fluminensis & Herb & 475 & 0.964 & 0.963 & 42.298 & 29.712 & 22.070 & 5.920 \\
Trapa natans & Aquatic Plant & 338 & 0.953 & 0.948 & 30.108 & 14.523 & 28.319 & 27.051 \\
Triadica sebifera & Tree & 197 & 0.969 & 0.966 & 33.714 & 22.678 & 42.117 & 1.492 \\
Trifolium dubium & Herb & 6,134 & 0.810 & 0.810 & 44.839 & 31.054 & 21.430 & 2.676 \\
Trifolium repens & Herb & 13,907 & 0.701 & 0.701 & 45.356 & 24.880 & 26.153 & 3.612 \\
Tussilago farfara & Herb & 8,610 & 0.768 & 0.768 & 43.446 & 19.040 & 30.313 & 7.201 \\
Typha latifolia & Aquatic Plant & 7,026 & 0.780 & 0.779 & 61.827 & 13.267 & 21.506 & 3.400 \\
Ulex europaeus & Tree & 3,749 & 0.867 & 0.867 & 35.517 & 39.869 & 16.012 & 8.602 \\
Urochloa mutica & Herb & 308 & 0.916 & 0.912 & 29.555 & 45.516 & 22.176 & 2.754 \\
Utricularia gibba & Aquatic Plant & 785 & 0.885 & 0.882 & 28.791 & 10.270 & 57.747 & 3.192 \\
Vallisneria nana & Aquatic Plant & 216 & 0.952 & 0.947 & 42.887 & 33.928 & 16.741 & 6.444 \\
Vallisneria spiralis & Aquatic Plant & 240 & 0.954 & 0.950 & 21.384 & 21.180 & 16.608 & 40.828 \\
\hline & & & & & & & &
\end{tabular}




\begin{tabular}{llrrrrrrr}
\hline Verbascum thapsus & Herb & 6,083 & 0.803 & 0.803 & 53.785 & 22.179 & 21.708 & 2.328 \\
Verbena brasiliensis & Herb & 185 & 0.975 & 0.973 & 65.427 & 8.170 & 21.179 & 5.224 \\
Verbena rigida & Herb & 560 & 0.962 & 0.961 & 37.398 & 27.507 & 28.530 & 6.565 \\
Vinca major & Herb & 2,432 & 0.899 & 0.898 & 44.897 & 30.663 & 13.815 & 10.624 \\
Vitex rotundifolia & Shrub & 196 & 0.967 & 0.964 & 18.553 & 19.162 & 58.350 & 3.935 \\
Vulpia bromoides & Herb & 4,725 & 0.836 & 0.835 & 54.048 & 42.092 & 2.658 & 1.202 \\
Waterhousea floribunda & Tree & 129 & 0.992 & 0.991 & 19.593 & 36.601 & 33.202 & 10.605 \\
Wisteria floribunda & Vine & 144 & 0.983 & 0.981 & 28.080 & 21.493 & 48.426 & 2.002 \\
Wisteria sinensis & Vine & 159 & 0.966 & 0.962 & 42.912 & 18.471 & 14.567 & 24.050 \\
Xanthium spinosum & Herb & 1,932 & 0.899 & 0.898 & 48.147 & 40.940 & 8.788 & 2.126 \\
Zantedeschia aethiopica & Herb & 464 & 0.964 & 0.963 & 41.649 & 51.710 & 5.889 & 0.752 \\
Zizania latifolia & Herb & 115 & 0.986 & 0.984 & 23.389 & 20.725 & 52.030 & 3.855 \\
Ziziphus mauritiana & Tree & 275 & 0.915 & 0.909 & 66.189 & 9.388 & 17.199 & 7.224 \\
Mean & & 1,864 & 0.911 & 0.909 & 38.442 & 29.915 & 25.360 & 6.283 \\
\hline
\end{tabular}

